\title{
Sistema para Rehabilitación del Síndrome del Miembro Fantasma utilizando Interfaz Cerebro- Computador y Realidad Aumentada
}

\author{
Juan Esteban Arango ${ }^{1}$, Julián Cárdenas $\mathrm{Mazo}^{1}$, Alejandro PeñaPalacio ${ }^{1}$ \\ juaross71@eia.edu.co, alcamaz62@eia.edu.co,pfjapena@eia.edu.co \\ ${ }^{1}$ Escuela de Ingeniería de Antioquia-Grupo GISMOC, 055428, Envigado, Colombia.
}

DOI: $10.4304 /$ risti.11.93-106

\begin{abstract}
Resumen: En el presente trabajo, se utilizó un dispositivo BCI comercial, el Emotiv EPOC, el cual es un neuroheadset inalámbrico de alta resolución para la adquisición de señales EEG, para desarrollar una herramienta con detección inteligente de patrones neuronales paralela a la del desarrollador para la implementación de una aplicación que combina la Realidad Aumentada (AR). La aplicación pensada como posible tratamiento del Dolor del Miembro Fantasma (PLP) en pacientes amputados. El desarrollo del motor de clasificación permitió tener un mayor control sobre los parámetros del procesamiento y detección de patrones en las señales, donde se obtuvo hasta un $82.1 \%$ de clasificación. Estas señales neuronales detectadas de un sujeto, se utilizan para descifrar su intención de cerrar o abrir un modelo virtual de una mano o de una prótesis adherida al muñón real a través del entorno $\mathrm{AR}$, brindando retroalimentación visual al paciente. Lo que contribuiría a reducir neurológicamente el PLP.
\end{abstract}

Palabras-clave: Interfaz Cerebro-Computador; Realidad Aumentada; Rehabilitación; PLP; Reconocimiento Inteligente de Patrones.

\begin{abstract}
In this study, we used a commercial BCI device, the Emotiv EPOC, which is a high-resolution wireless neuroheadset for EEG signal acquisition, to develop a intelligent pattern detection tool parallel to the developer's one to implement an application that combines Augmented Reality (AR). The application was developed as a possible treatment of phantom limb pain (PLP) in amputees patients. The classification engine development has allowed greater control over processing parameters and signal pattern detection, which yielded up to $82.1 \%$ of classification. These detected neural signals from a subject, were used to decode its intention of closing or opening a virtual model of a hand or prosthesis attached to the real stump through the AR environment, providing visual feedback to the patient. This, would neurologically help reduce the PLP.
\end{abstract}

Key-words: Brain-Computer Interface; Augmented Reality; Rehabilitation; PLP; Intelligent Pattern Recognition. 


\section{Introducción}

El síndrome del miembro fantasma, surge entre las personas que han sufrido una amputación de uno de sus miembros. Tras este suceso, la persona tiende a tener la sensación de aún contar con su miembro ausente (Flor et al., 1995). Este síndrome por lo general acarrea tres síntomas: Sensación del miembro fantasma, dolor del muñón y dolor del miembro fantasma. El primer síntoma se da en casi todos los casos y es raramente un problema clínico, el segundo síntoma recae en el proceso de curación del munón; mientras que un tercer síntoma, se centra en el del dolor del miembro fantasma, el cual es el más difícil de tratar. Este problema, se genera porque la persona amputada presenta sensaciones somato-sensoriales de que su miembro ausente está allí y está paralizado, y ante la necesidad de moverlo para dar a entender a su cerebro que efectivamente cuenta con él, desarrollan síntomas de ansiedad ante la incapacidad de hacerlo. Esto de forma crónica y de forma repetida, genera dolor en las personas.

Hay estudios que reportan que cerca del $60-80 \%$ de las personas con amputación presentan el dolor del miembro fantasma en algún momento (Nikolajsen \& Jensen, 2001), mientras otros reportan que el porcentaje real es de menos del 10\% ya que hay una correlación alta entre la cantidad de personas y su edad, pues aunque no se conoce a ciencia exacta su origen, proponen que el dolor se debe dar por la organización de las neuronas en la corteza motora del cerebro y entre más edad tenga la persona más organizada tiene una estructura nerviosa en su cerebro dedicada al uso de dicho miembro, y menor es la plasticidad cerebral para reemplazar el uso esas neuronas (Flor et al., 1995).

Las Interfaces Cerebro-Computador por su parte, son sistemas que decodifican las señales neuronales generadas intencionalmente por un sujeto, para traducirlas en órdenes para el computador, con el fin de conducir un actuador. Aunque no es una tecnología nueva, en los últimos años ha evolucionado a pasos agigantados (Martinovic et al., 2012; Sanchez \& Principe, 2007; Wu, Chabanon, Heidelberger, Li, \& Wang, 2012). Entre los diferentes enfoques que se le ha dado a estos sistemas, se han hecho avances desde la comprensión de las funciones cognitivas, hasta la conexión humana con entornos reales y virtuales a partir del pensamiento. En la decodificación del motor imagery a partir de las señales de EEG, se han desarrollado varias aplicaciones para el control de prótesis motoras, vehículos, entre otros (Hazrati \& Erfanian, 2010; Sancho Rieger et al., 2008; Schwartz, Cui, Weber, \& Moran, 2006).

En la literatura se pueden encontrar varios enfoques para el tratamiento de este síndrome complejo. Uno de los tratamientos no médicos utilizados por muchos terapeutas consiste en el principio de Bio-feedback (Nikolajsen \& Jensen, 2001). El más popular de este tipo de terapia es la caja-espejo creado por Ramachandran, el cual consiste en colocar un espejo en el medio de una caja, donde el paciente introduce ambos miembros: el amputado y el sano, en la cual el paciente moviendo la mano logra crear la ilusión en el espejo que su extremidad ausente se está moviendo simultáneamente (Ramachandran \& Rogers-Ramachandran, 1996). Los resultados han mostrado que este tratamiento ayuda a reducir significativamente la sensación del miembro fantasma y en consecuencia su dolor. Otros autores han utilizado tecnologías de realidad virtual y aumentada con el mismo principio, utilizando sensores en el miembro sano para crear la ilusión de movimiento del miembro opuesto, dando la 
información visual al cerebro que el movimiento de la extremidad sana es la misma del miembro fantasma (Cole, Crowle, Austwick, \& Slater, 2009; Desmond, O’Neill, De Paor, McDarby, \& MacLachlan, 2006; Mcgregor \& Dixon, 2011).

Este trabajo se centra en el desarrollo de una aplicación de alto impacto utilizando un sistema BCI portátil y asequible, combinado con una aplicación de realidad aumentada orientada a resolver un problema específico en rehabilitación de un síndrome común que la mayoría de los pacientes con amputaciones de extremidades sufren. El enfoque propuesto en este trabajo, difiere de otros en que el movimiento de la extremidad que falta no es controlado por otro elemento del cuerpo, más sí por sus propias señales neurofisiológicas moduladas por sus pensamientos, tal como se genera en un miembro sano, a partir del neuroheadset utilizado. El sistema posee una interacción del sujeto con la realidad aumentada y un sistema de calificación de estas señales que representan la sensación de movimiento en la prótesis virtual desarrollada.El desarrollo del motor de clasificación permitió tener un mayor control sobre los parámetros del procesamiento y detección de patrones en las señales, donde se obtuvo hasta un $82.1 \%$ de clasificación de los patrones generados con intención de movimiento. Estas señales neuronales detectadas de un sujeto, se utilizan para descifrar su intención de cerrar o abrir un modelo virtual de una mano o de una prótesis adherida al muñón real a través del entorno AR, brindando retroalimentación visual al paciente. Lo que contribuiría a reducir neurológicamente el PLP.

\section{Metodología}

Para llevar a cabo el sistema de rehabilitación mediante la utilización de interfaces BCI y realidad aumentada, se llevó a cabo en primer lugar un proceso de selección riguroso para determinar cuál era el mejor sistema para la adquisición de señales EEG entre una serie de productos comerciales disponibles utilizados para tal fin, de modo que cumplan con las necesidades del proyecto. En una siguiente etapa, o etapa de reconocimiento y de apropiación del equipo en cuanto a la formación de patrones mentales necesarios para controlar una aplicación. La facilidad y eficacia del sistema se midió en un primer acercamiento utilizando distintos sujetos siguiendo un protocolo de prueba. Después de ello, se propuso una integración de la BCI con una aplicación de realidad aumentada, con el fin de tratar de forma efectiva y alternativa el síndrome de dolor del miembro fantasma.

\subsection{Sistema de Adquisición de señales EEG}

Para la adquisición de las señales EEG, se realizó la selección de un sistema a partir de los sistemas actuales de adquisición de señales EEG. Comercialmente existen varios tipos de hardware con características diferentes, que se ajustan a distintas necesidades y aplicaciones. Entre los más representativos del mercado se destacan cuatro de ellos debido a su calidad, precio y popularidad en el campo de la investigación académica. Los productos pre-seleccionados fueron: g.BCIsys con g.USBamp de g.tec (G.tec, 2011) producidos en Austria, EEG Powerlab Data Adquisition System (ADInstruments, 2012) producido en Nueva Zelanda, Emotiv EPOC EEG (Emotiv, 2012) producido en Australia y Neurosky Mindwave (Neurosky, 2012) producido en Estados Unidos. 
Los criterios de evaluación para estos sistemas, se enfocaron en su capacidad, asequibilidad en el mercado, resolución, portabilidad, muestreo, compatibilidad, fiabilidad, flexibilidad y escalabilidad, entregando a cada criterio un peso específico asignado subjetivamente según las necesidades particulares del proyecto (ver Tabla 1).

Tabla 1 - Criterios de Evaluación para la Selección del Sistema de Adquisición

\begin{tabular}{ll}
\hline \multicolumn{1}{c}{ Criterios } & \multicolumn{1}{c}{ Detalle } \\
\hline Capacidad & Cantidad de canales de adquisición. \\
Asequibilidad & Precio, Distribución local, Promoción \\
Portabilidad & Usabilidad, alámbrico/inalámbrico, tipos de electrodos \\
Resolución & Espacial y el número de bits de la señal de lectura. \\
Muestreo & Frecuencia de adquisición, amplificación y filtrado \\
Compatibilidad & Facilidad de integración con distintas plataformas de software. \\
Fiabilidad & Marca, garantía, trabajos realizados, referencias \\
Flexibilidad & Tipo de sensores y señales que puede adquirir \\
& Librerías y funcionalidades que permitan procesar en \\
Escalabilidad & diferentes etapas las señales, ya sean desde su obtención como \\
& señales puras hasta ya clasificadas. \\
\hline
\end{tabular}

Las cuatro alternativas se clasificaron con base a estos parámetros, utilizando para ello un sistema de calificación de o a 5 puntos como se muestra en la Tabla 2 y la Figura 1. De esta etapa, el Emotiv EPOC (Adelson, 2011) fue clasificado como el mejor dispositivo, ya que este cumplía ampliamente con los requisitos mínimos para el desarrollo de este trabajo, por lo que se eligió para el desarrollo de la aplicación.

Tabla 2 - Evaluación de Sistemas de Adquisición EEG

\begin{tabular}{lrcccc}
\hline \multirow{2}{*}{ Criterios } & & \multicolumn{4}{c}{ Alternativas de Equipos } \\
\cline { 3 - 6 } & Pesos & g.USBamp & Powerlab & EmotivEPOC & Neurosky \\
\hline Capacidad & $10 \%$ & 5,0 & 5,0 & 4,0 & 1,0 \\
Asequibilidad & $20 \%$ & $\mathbf{1 , 0}$ & $\mathbf{1 , 0}$ & 4,0 & 5,0 \\
Portabilidad & $18 \%$ & $\mathbf{1 , 0}$ & 1,0 & 4,5 & 5,0 \\
Resolución & $5 \%$ & 5,0 & 4,5 & 4,5 & 5,0 \\
Muestreo & $5 \%$ & 5,0 & 4,5 & 3,5 & 3,0 \\
Compatibilidad & $15 \%$ & 4,5 & 2,0 & 4,5 & 5,0 \\
Fiabilidad & $10 \%$ & 5,0 & 4,5 & 5,0 & 4,5 \\
Flexibilidad & $5 \%$ & 5,0 & 5,0 & 4,0 & 2,0 \\
Escalabilidad & $12 \%$ & 5,0 & 1,0 & 5,0 & 5,0 \\
\hline Total Ponderado & $\mathbf{1 0 0} \%$ & $\mathbf{3 . 4 1}$ & $\mathbf{2 . 4 5}$ & $\mathbf{4 . 3 9}$ & $\mathbf{4 . 2 5}$ \\
\hline
\end{tabular}




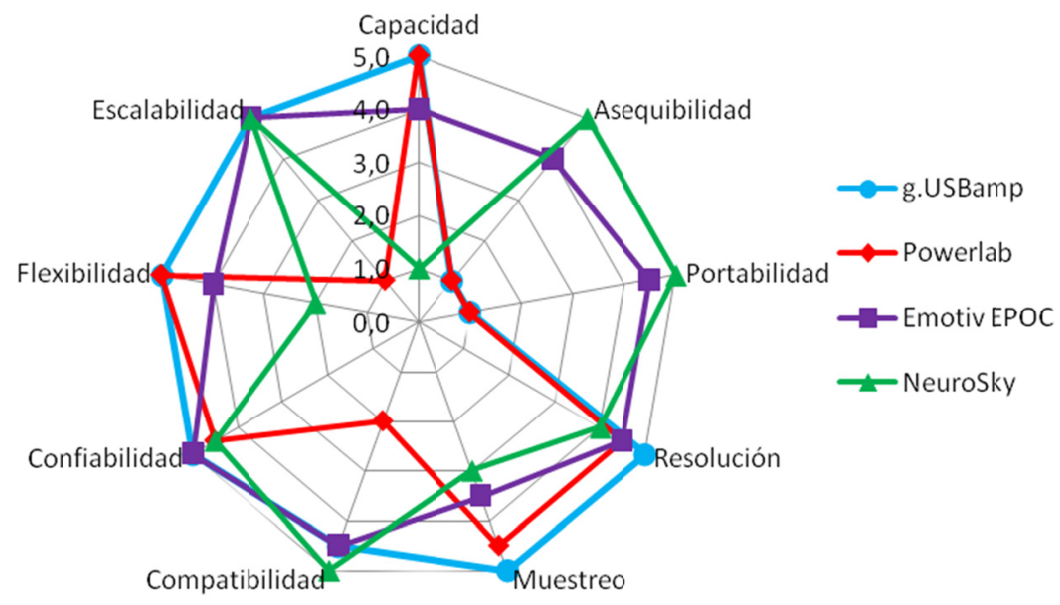

Figura 1 - Evaluación de Sistemas de Adquisición EEG

De lo anterior, se puede observar que el dispositivo Emotiv EPOC, es un neuroheadset inalámbrico de alta resolución para la adquisición y procesamiento de señales EEG. El registro se realiza a través de 14 canales correspondientes a 14 electrodos secos colocados en la parte superior de la cabeza de acuerdo al sistema 10-20 de posicionamiento de electrodos para EEG, ilustrado en la Figura 2.

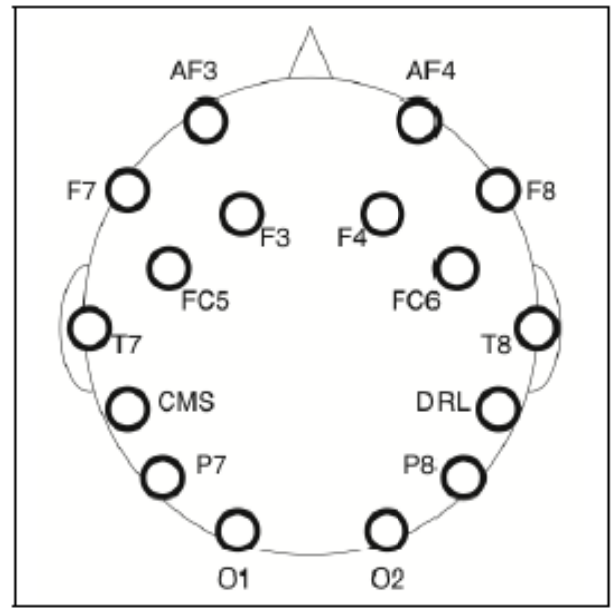

Figura 2 - Posicionamiento de los electrodos del Emotiv EPOC (Emotiv, 2012)

\subsection{Procesamiento de la señal}

En esta etapa, se realizó el procesamiento de señales a partir del dispositivo EEG seleccionado. Este sistema en su hardware y software, cuenta con unidades de pre- 
procesamiento y procesamiento para el reconocimiento de los diferentes estados o representaciones mentales del usuario. Este hardware posee tres filtros: un filtro pasa baja con un corte a $85 \mathrm{~Hz}$, un filtro pasa alta con un punto de corte a $0,16 \mathrm{~Hz}$ y un filtro rechaza bandas en 50 y 6o Hz. Su tasa de muestreo es de $2048 \mathrm{~Hz}$, pero se reduce a 128 $\mathrm{Hz}$ para la transmisión inalámbrica. El software consta de tres suites de detección, que se detallan en la Tabla 3. El neuroheadset también tiene un API (Application Programming Interface) para desarrolladores, la cual permite programar eventos (llamados EmoEvents) en base a los que patrones o estados que se detecten (EmoStates). Estos estados o representaciones mentales reflejan los gestos del usuario, las emociones y los pensamientos.

Para el funcionamiento del Emotiv, un usuario debe realizar un proceso de entrenamiento para reproducir y controlar determinados patrones o EmoStates que se utilizan como comandos en diferentes aplicaciones como cursor de mouse, teclados deletreadores o juegos. Este entrenamiento es obligatorio para cada usuario y su dificultad varía particularmente entre usuarios.

Tabla 3 - Suites de detección y estados que permite detectar el Emotiv EPOC

\begin{tabular}{c|l|l}
\hline Suite & Descripción & Estados (EmoStates) \\
\hline \multirow{2}{*}{ Expressiv } & $\begin{array}{c}\text { Permite detectar gestos } \\
\text { faciales. }\end{array}$ & $\begin{array}{l}\text { Blink, Wink Left, Wink Right, Look Left, Look Right, } \\
\text { Eyebrow Raised, Furrow, Smile, Clench, Laugh, Smirk } \\
\text { Left, Smirk Right }\end{array}$ \\
Affectiv & $\begin{array}{c}\text { Permite detectar estados } \\
\text { emocionales del sujeto }\end{array}$ & $\begin{array}{l}\text { Excitement Short Term, Excitement Long Term, } \\
\text { Engagement Boredom }\end{array}$ \\
& $\begin{array}{c}\text { Permite detectar estados } \\
\text { cognitivos a partir de los } \\
\text { pensamientos del sujeto }\end{array}$ & $\begin{array}{l}\text { Neutral, Push, Pull, Lift, Drop, Left, Right, Rotate Left, } \\
\text { R. Right, R. Clockwise, R. Counterclockwise, R. } \\
\text { Forward, R. Backward, Disappear }\end{array}$ \\
\hline
\end{tabular}

\subsection{Caracterización del sistema}

El dispositivo Emotiv está fabricado como una plataforma cerrada, la cual permite desarrollar aplicaciones para manipular señales EEG sin requerir de conocimientos previos en neurociencias, fisiología o ingeniería. Para un funcionamiento más avanzado, el usuario de este dispositivo solo requiere de conocimientos básicos de programación para acceder a los estados de las suites de detección, mediante la API que el fabricante pone a disposición. Por esta razón se procedió a realizar una caracterización del sistema en el modelo de detección de estados cognitivos. Esta caracterización se realizó utilizando máquinas de vector soporte (SVM) sobre patrones espaciales comunes (CSP) (Arango Ossa \& Cárdenas Mazo, 2012). Aunque la caracterización solo permite hasta el momento el reconocimiento de dos estados cognitivos off-line, esta aproximación permitirá en un futuro poder desarrollar un algoritmo que permita la detección de múltiples estados cognitivos, y tener la libertad de implementarlo en plataformas móviles. 


\subsection{Diseño de Experimentos}

Para el diseño de experimentos, se planteó un protocolo de pruebas para examinar la capacidad y usabilidad del BCI por diferentes usuarios, y así mismo para obtener datos importantes acerca de las variables que pueden afectar el rendimiento del sistema. Seis sujetos entre ellos hombres y mujeres diestros (20-25 años de edad) participaron en este estudio. Este número fue identificado por diferentes investigadores, los cuales han obtenido resultados interesantes al realizar experimentos con esta cantidad de participantes (Bradberry, Gentili, \& Contreras-Vidal, 2010; Waldert et al., 2008). Los sujetos para participar, firmaron un consentimiento informado. Las pruebas se realizaron siguiendo las recomendaciones formuladas en el BBCI Workshop: Advances in Neurotechnologies, Berlin 2009 (Müller \& Blankertz, 2009).

Las pruebas se dividieron en dos fases: en una primera fase, el sujeto fue entrenado durante diez minutos sin retroalimentación visual, y se le pidió representar mentalmente dos estados cognitivos: un estado neutral, y la imaginación del movimiento de cierre de la mano ayudado de un monitor que enseñaba una secuencia aleatoria que el sujeto debía reproducir. Durante este proceso, el motor del Emotiv llevo a cabo un proceso de aprendizaje sobre cada estado: neutral y cierre de la mano, este último asignado a una EmoState específico. En este punto, la capacidad del sujeto para replicar el estado solicitado fue evaluado, esto gracias a la retroalimentación visual de la prótesis virtual al moverse o no. Adicionalmente, se le solicitó al participante que entrenara durante 10-15 minutos el sistema, para que luego reprodujera una secuencia de 60 estados, esto con el fin de medir la tasa de éxito en el reconocimiento. En la segunda fase del experimento, la cual fue similar a la primera, en vez de dos estados se hizo con tres estados cognitivos: el estado neutral, la imaginación de cierre de la mano, y la imaginación de apertura de la mano. En esta etapa el entrenamiento se hizo entre 20 a 30 minutos.

\subsection{Integración para la Aplicaciones}

El objetivo de la realidad aumentada (AR), consiste en aumentar la percepción de lo que realmente se ve, mediante la adición de modelos virtuales $3 \mathrm{D}$ integrados al entorno real que se reproduce a través de dispositivos de visualización. Hay varios programas desarrollados que permiten la creación de aplicaciones de AR, como ARToolKit, ARmedia, SLARTToolkit y D'Fusion Studio para nombrar unos pocos. Para el caso particular de este trabajo, se optó por la utilización del D'Fusion Studio de Total Immersion, esto gracias a su robustez, flexibilidad y licenciamiento gratuito para uso no comercial.

El funcionamiento de esta aplicación se muestra en la Figura 3, en donde los patrones entrenados por el usuario de la BCI son la entrada a la interfaz de AR, y cada patrón se convierte en un comando para controlar el movimiento de la mano virtual. Modelo que se superpone sobre el muñón del paciente amputado, dando la ilusión de que la mano virtual modelada pertenece realmente al paciente. 


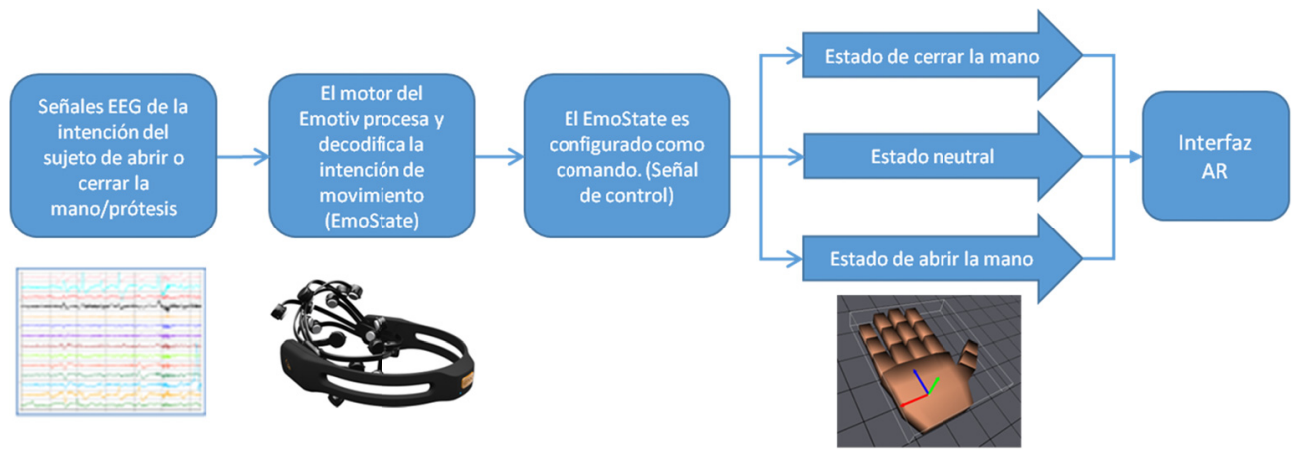

Figura 3 - Diagrama del sistema integrado del equipo BCI con el entorno AR.

\subsection{Realidad Aumentada}

En un típico esquema de AR, hay algunos elementos esenciales. En primer lugar, se necesita un dispositivo de captura de vídeo para grabar el entorno real; aquí, la cámara y la iluminación son muy importantes. Para la captura del video, se utilizó una cámara inalámbrica móvil de alta definición, y un entorno de iluminación controlado utilizando lámparas incandescentes habituales de laboratorio. La forma en que los modelos reales y virtuales interactúan, es por medio del reconocimiento de imágenes de marcadores específicos en el entorno de grabación. Estos marcadores pueden ser códigos bidimensionales a blanco y negro, como un código $\mathrm{QR}$, o imágenes muy características con texturas. Para el caso particular de este proyecto, el interés se centró en el reconocimiento del muñón de un paciente amputado, razón por la cual se diseñó un calcetín especial que cualquier paciente pudiera vestir y que el software lograra identificar. Para tal efecto, se probaron imágenes de calcetines con diferentes texturas. Para la representación del muñón, se realizó un molde de yeso de un antebrazo distal amputado para utilizar en la etapa de desarrollo (véase la Fig. 4). El modelo virtual 3D desarrollado para esta aplicación, fue una mano humana modelada y animada en software CAD (ver Fig. 5).
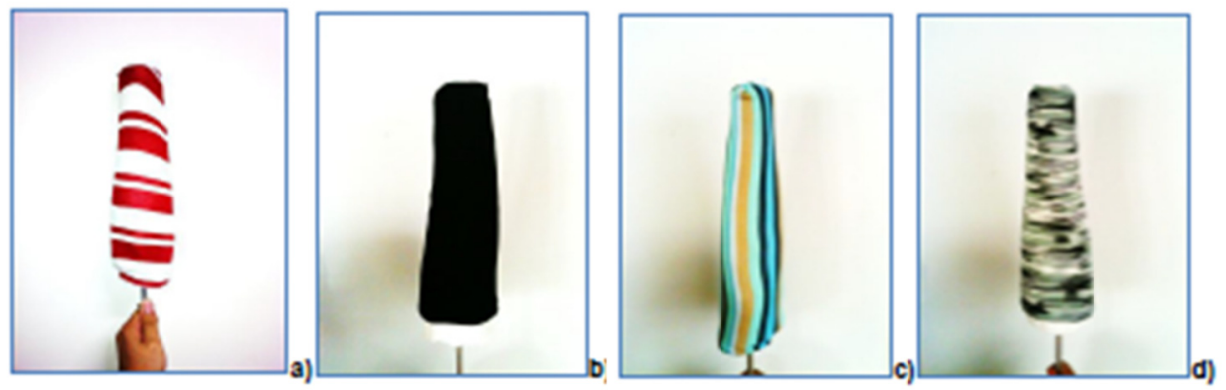

Figura 4 - Marcadores para el entorno AR. Medias con diferentes texturas sobre un modelo en yeso del muñón de un brazo con amputación. 

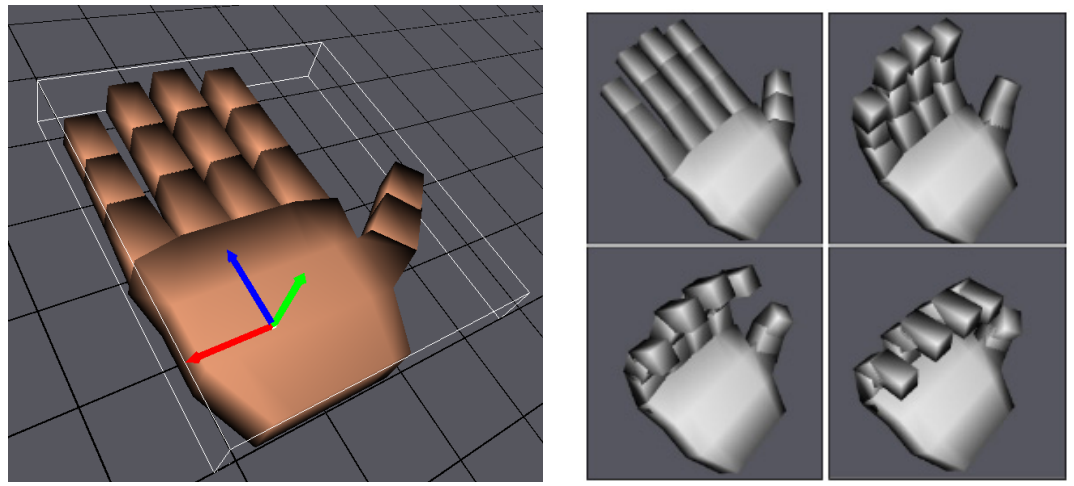

Figura 5 - Modelo 3D de una mano. Con simulación de movimiento de abrir y cerrar.

\section{Resultados}

Para lograr que un paciente manipule su miembro fantasma, en este caso, que logre manipular el modelo virtual que el sistema de realidad aumentada le muestra como parte de su propio cuerpo, necesita varias horas de entrenamiento para controlar los patrones que representan cada movimiento en términos de las señales EEG, tal como lo evidencian los experimentos. Como se muestra en la Figura 6a, con sólo 20 minutos de entrenamiento, un usuario está en capacidad de controlar dos estados diferentes, la representación neutral y de otro tipo, con un promedio de 80,5\% de precisión. Para el caso de varios estados, como en el caso del entrenamiento para tres estados diferentes, representaciones neutrales y dos más, el nivel de precisión se redujo significativamente (Ver Fig. 6b). Sólo 2 de 6 usuarios que utilizaron la BCI, lograron controlar el movimiento con una precisión cercana al 100\%, pero los otros 4 fallaron en el intento.
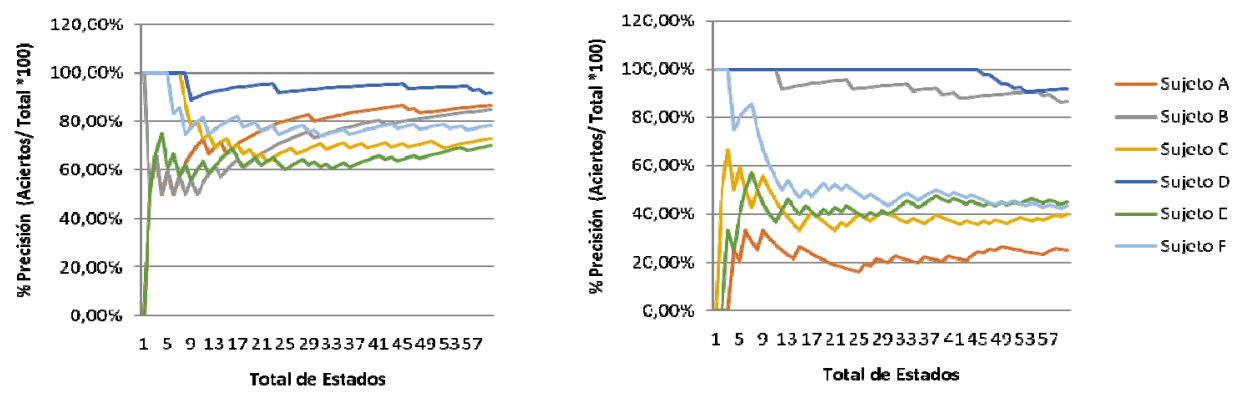

Figura 6 - Resultados del experimento realizado con 6 sujetos. a) Manejo de un sólo Estado. b) Manejo de dos Estados.

De esta manera, se pudo evidenciar que la eficacia y facilidad de uso del sistema depende de diferentes variables entre las que se cuentan: la cantidad de estados entrenados, entre mayor cantidad de estados que el usuario desee controlar, más difícil es su uso; la capacidad de cada usuario de crear mentalmente una representación totalmente exclusiva de otra; el nivel de concentración en donde el usuario debe estar enfocado en utilizar el BCI; y la frustración, la cual en un principio es difícil de usar y 
puede generar episodios de estrés en el paciente y disminuir la precisión que se requiere para el reconocimiento.

Para la integración con la tecnología de Realidad Aumentada se probaron diferentes marcadores, utilizando para ello cuatro diferentes tipos de calcetines. Cada calcetín aportaba una textura diferente al algoritmo de reconocimiento de imágenes del software de AR. Los mejores resultados se obtuvieron con el calcetín que se muestra en la figura 4d. Con éste, es posible obtener una detección más flexible y potente del muñón del paciente amputado o el modelo de yeso para la prueba en este caso.

Para proporcionar funcionalidad al sistema, cada sujeto en particular realizó un proceso de entrenamiento de las representaciones mentales hasta alcanzar un dominio de los patrones que pudiera reproducir fielmente, estos patrones se configuraron para ser las entradas al mecanismo propuesto. Los resultados del modelo son un sistema que brinda retroalimentación visual del miembro amputado a los usuarios, de una forma interactiva y similar al movimiento natural. Dicho bio-feedback ha sido crucial en terapias de reducción del dolor del miembro fantasma (Chan et al., 2007). Es por esto que el sistema desarrollado se propone como tratamiento de este complejo síndrome. Además, este sistema como tratamiento proporciona información visual de un miembro amputado artificial, que a diferencia de los métodos convencionales no requiere que la extremidad opuesta esté sana y funcional, pues el dominio del movimiento se hace a través de los estados cognitivos.
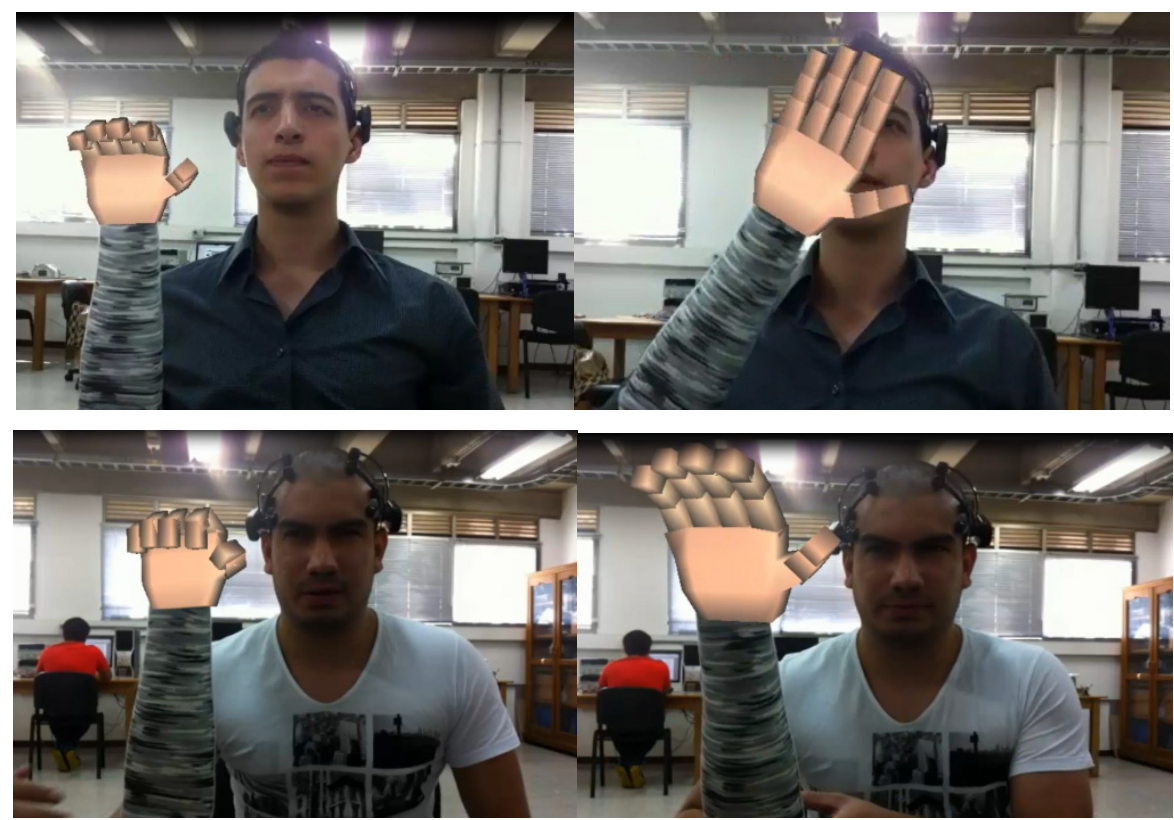

Figura 7 - Demostración del sistema funcional.

En la figura 7, se enseña cómo funciona la aplicación mediante una documentación fotográfica del sistema, y se evidencia cómo los resultados son positivos. En el producto final, el usuario puede ver recreada virtualmente, en tres dimensiones y en tiempo real 
su mano amputada, consiguiendo además controlarla voluntariamente con la modulación de sus pensamientos.

\section{Discusión}

En este trabajo se presenta un posible tratamiento para el dolor del miembro fantasma en personas con amputación de miembros. La solución propuesta integra una interfaz cerebro-computador, que traduce las representaciones mentales en comandos de control para una prótesis virtual, y una interfaz de AR a cargo de la visualización y la animación de la prótesis virtual. Esto permite que un sujeto tenga un control cognitivo de dicha prótesis, y una retroalimentación visual más real de movimiento de su extremidad ausente. Es un sistema interactivo que tiene potencial de uso en clínicas, hospitales y laboratorios, e incluso dada su portabilidad, este puede ser utilizado como sistema de rehabilitación en el hogar. Igualmente, este desarrollo arrojó como resultado una herramienta para entrenar a un sujeto que se encuentre en proceso de utilizar una verdadera prótesis neural con o sin BCI, configurando la entrada real en este software.

El sistema desarrollado puede ser utilizado para reconocer el muñón por su geometría en lugar de una imagen de la textura de la media. Por su parte, el tratamiento se puede adaptar para utilizarlo en amputaciones de extremidades inferiores, mientras que el modelo virtual, puede ser más realista mediante el uso de texturas que puedan replicar el color de la piel, las uñas, y los poros, y tienen la posibilidad de ser utilizados en pacientes con diferentes colores de piel.

En la etapa de diseño y desarrollo de la aplicación, que abarca múltiples campos del conocimiento, permitirá que en trabajos futuros se pueda validar el tratamiento, llevando a cabo experimentos con pacientes reales con extremidades amputadas, utilizando para ello métricas objetivas que permitan cuantificar la reducción en la sensación y el dolor en los pacientes con síndrome del miembro fantasma.

\section{Conclusiones}

De acuerdo con la metodología propuesta, se obtuvo un sistema en el que un paciente con amputación de uno de sus miembros puede aprender a manipular a intención una prótesis virtual con la ayuda de la BCI, lo que consigue transmitir a sus sentidos la sensación de que el modelo simulado es parte de sí mismo gracias al uso de la tecnología AR. Esto, con el fin de lograr que el movimiento sea voluntario y natural.

Los resultados de los experimentos, mostraron que el sistema de rehabilitación propuesto es relativamente fácil de utilizar por personas sanas, con tiempos de entrenamiento que rondan los 30 minutos. Para estudios futuros esto se puede corroborar con un tiempo de entrenamiento mayor, y el posible tratamiento tiene que ser probado en personas que realmente sufren del dolor del miembro fantasma.

Este tratamiento se puede extender a pacientes con amputación o discapacidad motora de la extremidad opuesta, ya sea en los miembros superior o inferior, a diferencia de los tratamientos actuales, ya que los tratamientos existentes, requieren que la extremidad opuesta esté saludable para recrear el movimiento de la extremidad fantasma. En el 
caso de la caja espejo, la extremidad libre de amputación ayuda a conseguir una imagen especular óptica, mientras que en el caso de terapias por realidad virtual y aumentada, se utiliza comúnmente un guante especial que transmite el movimiento a la extremidad virtual.

Esta es la primera aplicación desarrollada que combina estas dos tecnologías emergentes, la Interfaz Cerebro Computador y Realidad Aumentada en el campo de la medicina y la salud, ya que la experiencia más cercana en este tipo de sistemas, es utilizada para guiar la navegación de robots (Kansaku, Hata, \& Takano, 2010).

El uso de estas dos tecnologías por separado o en conjunto, tienen gran potencial de contribución en aplicaciones dirigidas a ayudar a las personas en situación de discapacidad, como una herramienta de accesibilidad. Para la rehabilitación, la comunicación, el uso de un ordenador o de diferentes dispositivos externos.

Este trabajo es un acercamiento interesante a las terapias de rehabilitación de nueva generación, en las que se utilicen herramientas de interfaces interactivas que faciliten a los pacientes interactuar activamente con los sistemas ya sea para diagnóstico, terapia o entretenimiento. En la medida en que se optimice esta comunicación, mejores dispositivos y aplicaciones surgirán en el proceso.

\section{Referencias bibliográficas}

Adelson, M. (Princeton U. (2011). Emotiv Experimenter. An experimentation and mind-reading application for the Emotiv EPOC. Princeton. Retrieved from http://compmem.princeton.edu/experimenter/

ADInstruments. (2012). About ADInstruments | ADInstruments. Retrieved from http://www.adinstruments.com/company/about

Arango Ossa, J. E., \& Cárdenas Mazo, J. (2012). ALGORITMO INTELIGENTE PARA MANIPULACIÓN DE ACTUADORES POR MEDIO DE INTERFAZ CEREBRO COMPUTADOR ( $B C I$ ). Escuela de Ingeniería de Antioquia.

Bradberry, T. J., Gentili, R. J., \& Contreras-Vidal, J. L. (2010). Reconstructing threedimensional hand movements from noninvasive electroencephalographic signals. The Journal of neuroscience : the official journal of the Society for Neuroscience, 3O(9), 3432-7. doi:10.1523/JNEUROSCI.6107-09.2010

Chan, B. L., Witt, R., Charrow, A. P., Magee, A., Howard, R., Pasquina, P. F., Heilman, K. M., et al. (2007). Mirror therapy for phantom limb pain. The New England journal of medicine, 357(21), 2206-7. doi:10.1056/NEJMc071927

Cole, J., Crowle, S., Austwick, G., \& Slater, D. H. (2009). Exploratory findings with virtual reality for phantom limb pain; from stump motion to agency and analgesia. Disability and rehabilitation, 31(10), 846-54. doi:10.1080/09638280802355197.

Desmond, D. M., O’Neill, K., De Paor, A., McDarby, G., \& MacLachlan, M. (2006). Augmenting the Reality of Phantom Limbs: Three Case Studies Using an Augmented Mirror Box Procedure. JPO Journal of Prosthetics and Orthotics, 18(3), 74-79. doi:10.1097/00008526-200607000-00005 
Emotiv. (2012). Emotiv Software Development Kit. Retrieved from http://emotiv.com/developer/SDK/UserManual.pdf

Flor, H., Elbert, T., Knecht, S., Wienbruch, C., Pantev, C., Birbaumer, N., Larbig, W., et al. (1995). Phantom-limb pain as a perceptual correlate of cortical reorganization following arm amputation. Nature, 375(6531), 482-4. doi:10.1038/375482ao

G.tec. (2011). Products Catalogue. G.Tec. Retrieved from http://www.gtec.at/Download/Product-Brochures/g.tec-Product-Catalogue

Hazrati, M. K., \& Erfanian, A. (2010). An online EEG-based brain-computer interface for controlling hand grasp using an adaptive probabilistic neural network. Medical engineering \& physics, 32(7), 730-9. doi:10.1016/j.medengphy.2010.04.016

Kansaku, K., Hata, N., \& Takano, K. (2010). My thoughts through a robot's eyes: an augmented reality-brain-machine interface. Neuroscience research, 66(2), 21922. doi:10.1016/j.neures.2009.10.006

Martinovic, I., Davies, D., Frank, M., Perito, D., Ros, T., \& Song, D. (2012). On the Feasibility of Side-Channel Attacks with Brain-Computer Interfaces. Security'12 Proceedings of the 21st USENIX conference on Security symposium (pp. 34-34). California: USENIX Association Berkeley, CA, USA (C)2012.

Mcgregor, G., \& Dixon, B. (2011). From Mirror Therapy to Augmentation Holger Regenbrecht Elizabeth Franz Simon Hoermann The Information Science Discussion Paper Series.

Müller, K.-R., \& Blankertz, B. (2009). Machine Learning and Signal Processing Tools for BCI. BBCI Workshop: Advances in Neurotechnologies, Berlin 2009. Berlin. Retrieved from http://videolectures.net/bbciog_blankertz_muller_mlasp/

Neurosky. (2012). NeuroSky - Brainwave Technology. Retrieved from http://www.neurosky.com/

Nikolajsen, L., \& Jensen, T. S. (2001). Phantom limb pain. British journal of anaesthesia, 87(1), 107-16. Retrieved from http://www.ncbi.nlm.nih.gov/pubmed/22349560

Ramachandran, V. S., \& Rogers-Ramachandran, D. (1996). Synaesthesia in phantom limbs induced with mirrors. Proceedings. Biological sciences / The Royal Society, 263(1369), 377-86. doi:10.1098/rspb.1996.0058

Sanchez, J. C., \& Principe, J. C. (2007). Brain-Machine Interface Engineering. Synthesis Lectures on Biomedical Engineering (Vol. 2, pp. 1-234). doi:10.2200/Soo053ED1V01Y200710BME017

Sancho Rieger, J. (Junta D. de la S. E. de N. (SEN)), Waldert, S., Pistohl, T., Braun, C., Ball, T., Aertsen, A., Mehring, C., et al. (2008). Neural control of motor prostheses. Neuron, 32(1), 480-94. doi:10.1016/j.neuron.2008.10.037

Schwartz, A., Cui, X., Weber, D., \& Moran, D. (2006). Brain-Controlled Interfaces: Movement Restoration with Neural Prosthetics. Neuron, 52(1), 205-220. doi:10.1016/j.neuron.2006.09.019 
Waldert, S., Preissl, H., Demandt, E., Braun, C., Birbaumer, N., Aertsen, A., \& Mehring, C. (2008). Hand movement direction decoded from MEG and EEG. The Journal of neuroscience : the official journal of the Society for Neuroscience, 28(4), 1000-8. doi:10.1523/JNEUROSCI.5171-07.2008

Wu, M. M., Chabanon, B., Heidelberger, G., Li, S., \& Wang, A. (2012). Brain Control. NJ Governor's School of Engineering \& Technology 2012. 